\title{
Update on shedding and transmission routes of porcine haemotrophic mycoplasmas in naturally and experimentally infected pigs
}

Julia Ade ${ }^{2}$, Mathias Ritzmann ${ }^{1}$, Christopher Wöstmann', Matthias Eddicks', Sven Reese ${ }^{3}$, Katharina Hoelzle ${ }^{2}$, Ludwig E. Hoelzle ${ }^{2}$ and Julia Stadler ${ }^{1^{*}}$ (D)

\begin{abstract}
Horizontal transmission of Mycoplasma suis via parenteral exposure during standard practices or through bites during fightings have been identified as key epidemiological routes. However, as knowledge gaps on other potential shedding and transmission routes exist, the present study combines both laboratory experiments and field surveys to gain new insights into the epidemiology of porcine haemotrophic mycoplasmas. Splenectomised pigs were orally inoculated with a M. suis field strain and investigated for clinical signs related to infectious anaemia of pigs (IAP) and the presence of M. suis in blood, urine and saliva samples by qPCR. All blood samples were negative for M. suis and animals did not show obvious clinical signs of IAP throughout the entire study period. Additionally, urine, nasal and saliva samples from sows of conventional piglet producing farms and semen samples from a boar stud revealed no detection of M. suis and 'Candidatus Mycoplasma haemosuis' by qPCR. Thus, the results indicate that blood-independent transmission routes might be of minor relevance under field conditions.
\end{abstract}

Keywords: Porcine haemotrophic mycoplasmas, Mycoplasma suis, 'Candidatus Mycoplasma haemosuis', Oral inoculation, Shedding patterns

\section{Background}

Haemotrophic mycoplasmas (HMs) are uncultivable bacteria found on the surface of red blood cells (RBCs) of numerous domestic and wild mammals [1]. Mycoplasma suis, the mostly studied porcine HM species, is considered as the causative agent of infectious anaemia in pigs (IAP), causing important economic losses in pig production $[2,3]$. The disease can either occur as an acute, haemolytic anaemia attended by high fever and life-threatening conditions or as a chronic or even subclinical form of disease with mild to moderate anaemia and unspecific clinical signs [2, 3]. Recently, a novel HM

\footnotetext{
*Correspondence: j.stadler@med.vetmed.uni-muenchen.de

${ }^{1}$ Clinic for Swine, Centre for Clinical Veterinary Medicine, LMU Munich, Sonnenstr. 16, Oberschleissheim, 85764 Munich, Germany

Full list of author information is available at the end of the article
}

species currently named as 'Candidatus (Ca.) Mycoplasma haemosuis' was described in subclinical diseases as well as in accordance with IAP-like signs in pigs in China, Korea and Germany [4-6].

Natural routes of porcine HM transmission remain rather unknown [7-9]. Experimental transmission by intravenous, intramuscular, subcutaneous, intraperitoneal and oral inoculation of M. suis containing blood has been successfully performed $[7,10]$. However, oral infection experiments were conducted prior to the establishment of specific and sensitive PCR assays using microscopic methods [7]. Thus, the first objective of the study was to demonstrate the possibility of oral infection in experimentally infected pigs compared to a subcutaneous infected control group.

Recently, $M$. suis shedding was demonstrated in blood-free excretions (i.e. saliva, urine, nasal and vaginal

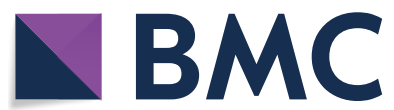

(c) The Author(s) 2021. Open Access This article is licensed under a Creative Commons Attribution 4.0 International License, which permits use, sharing, adaptation, distribution and reproduction in any medium or format, as long as you give appropriate credit to the original author(s) and the source, provide a link to the Creative Commons licence, and indicate if changes were made. The images or other third party material in this article are included in the article's Creative Commons licence, unless indicated otherwise in a credit line to the material. If material is not included in the article's Creative Commons licence and your intended use is not permitted by statutory regulation or exceeds the permitted use, you will need to obtain permission directly from the copyright holder. To view a copy of this licence, visit http://creativecommons.org/licenses/by/4.0/. The Creative Commons Public Domain Dedication waiver (http://creativeco mmons.org/publicdomain/zero/1.0/) applies to the data made available in this article, unless otherwise stated in a credit line to the data. 
secretion) [11] suggesting transmission of porcine HMs through direct contact via excretions. However, the investigated excretions originated from experimentally infected pigs with high blood loads [11]. Thus, the second aim of the study was to investigate shedding of porcine HMs ( $M$. suis and ' $C a$. M. haemosuis') via blood-independent excretions (saliva, urine and semen samples) under field conditions.

\section{Results}

\section{Clinical and pathological observations} during experimental $M$. suis infection

In the group of orally infected pigs (group A), clinical signs related to IAP were absent in all seven animals until the end of the study on 90 DPI and the clinical score remained at zero points for each animal. In contrast, all subcutaneously infected piglets (group B) showed typical signs of IAP as described by Stadler et al. [12]. Three animals (ID 23, 32, 76) developed fever, apathy, anorexia and skin alterations on 7 and 8 DPI. The determination criteria (i.e. high fever, anorexia, impaired general health) were reached on 8 DPI and the three pigs were humanely euthanized. The four remaining animals showed cyanoses of the ears between 13 and 15 DPI. Clinical IAP signs exacerbated in those four remaining animals and euthanasia had to be performed on 17 (ID 73), 20 (ID 74), 41 (ID 31) and 62 (ID 71) DPI, respectively. Clinical score points of group B animals (ID 23, 31, 32, 71, $73,74,76)$ are shown in Fig. 1. During necropsy, none of the seven orally infected pigs (group A) showed macroscopic alterations. Thus, no further microscopic investigation was conducted in those animals. Group B animals (subcutaneously infected) showed various macroscopic (i.e. e. severe icterus of membranes, yellowish discoloration of skin and body fluids, pale musculature) and histopathological lesions (i.e. hyaline thrombi and globules in alveolar vessels, haemosiderin deposits in macrophages, dilatation of lymph vessels, periportal and centrilobular necrosis) as described elsewhere [12].

\section{Detection of $M$. suis in blood, saliva and urine samples of experimentally infected pigs}

Mycoplasma suis qPCR remained negative for blood, urine and saliva samples of all group A piglets throughout the entire study period. In group B, positive M. suis results were obtained from the blood of all animals as described elsewhere [12]. Briefly, on 4 DPI M. suis was first detected in the blood of three animals (ID 23, 74, 76) and on 6 DPI in all seven animals of this group. Subsequently, M. suis was permanently present in the blood of group $B$ animals until the individual termination point of the study [12]. M. suis blood loads varied between $2.2 \times 10^{3}$ and $8.6 \times 10^{9}$ M. suis $/ \mathrm{mL}$ blood.

qPCR could also detect $M$. suis in the urine samples of two animals on 8 DPI (ID 73) and on 28 and 48 DPI (ID 71), respectively. $M$. suis loads in urine samples varied between $2.40 \times 10^{4}$ and $5.5 \times 10^{4}$ M. suis $/ \mathrm{mL}$ urine. In each of the three urine samples, Servotest ${ }^{\circledR} 5+\mathrm{NL}$ stripes showed the presence of RBC residues.

Regarding the saliva samples of group B animals, M. suis was evident in two animals on 8 DPI (ID 31+ ID 71 ), and on 15 DPI in one animal (ID 71). Bacterial loads in saliva samples varied between $2.0 \times 10^{3}$ and $5.26 \times 10^{3}$ M. suis $/ \mathrm{mL}$ saliva.

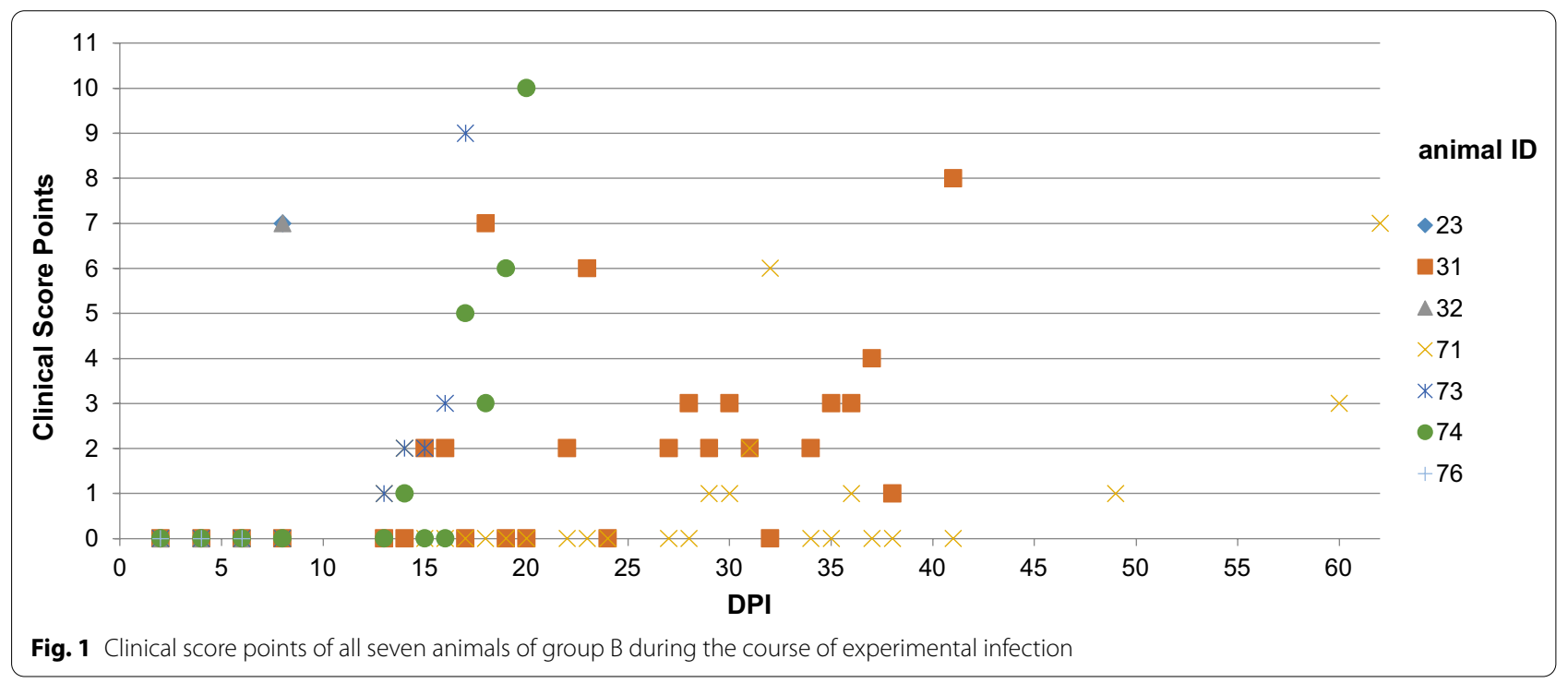


Table 1 Mycoplasma suis quantification of blood, urine and saliva samples determined by qPCR during experimental infection in group B animals (subcutaneously infected animals)

\begin{tabular}{|c|c|c|c|c|c|c|c|c|}
\hline \multirow{2}{*}{$\begin{array}{l}\text { Days post } \\
\text { infection }\end{array}$} & \multirow[t]{2}{*}{ M. suis $/ \mathrm{mL}$} & \multicolumn{7}{|c|}{ Animal ID (group B animal) } \\
\hline & & ID 23 & ID 31 & ID 32 & ID 71 & ID 73 & ID 74 & ID 76 \\
\hline \multirow[t]{3}{*}{2} & Blood & Negative & Negative & Negative & Negative & Negative & Negative & Negative \\
\hline & Urine & Negative & Negative & Negative & Negative & Negative & Negative & Negative \\
\hline & Saliva & Negative & Negative & Negative & Negative & Negative & Negative & Negative \\
\hline \multirow[t]{3}{*}{4} & Blood & $2.2 \times 10^{3}$ & Negative & Negative & Negative & Negative & $2.0 \times 10^{5}$ & $6.0 \times 10^{4}$ \\
\hline & Urine & Negative & Negative & Negative & Negative & Negative & negative & Negative \\
\hline & Saliva & Negative & Negative & Negative & Negative & Negative & Negative & Negative \\
\hline \multirow[t]{3}{*}{6} & Blood & $4.6 \times 10^{8}$ & $1.1 \times 10^{4}$ & $4.2 \times 10^{7}$ & $3.6 \times 10^{5}$ & $1.9 \times 10^{4}$ & $6.6 \times 10^{4}$ & $1.1 \times 10^{9}$ \\
\hline & Urine & Negative & Negative & Negative & Negative & Negative & Negative & Negative \\
\hline & Saliva & Negative & Negative & Negative & negative & Negative & Negative & Negative \\
\hline \multirow[t]{3}{*}{8} & Blood & $3.6 \times 10^{9} \dagger$ & $9.2 \times 10^{6}$ & $9.6 \times 10^{9} \dagger$ & $3.0 \times 10^{9}$ & $3.0 \times 10^{7}$ & $1.8 \times 10^{7}$ & $8.6 \times 10^{9} \dagger$ \\
\hline & Urine & Negative & Negative & Negative & Negative & $5.5 \times 10^{4}$ & Negative & Negative \\
\hline & Saliva & Negative & $2.0 \times 10^{3}$ & Negative & $2.84 \times 10^{3}$ & Negative & Negative & Negative \\
\hline \multirow[t]{3}{*}{14} & Blood & & $1.4 \times 10^{8}$ & & $1.9 \times 10^{7}$ & $3.6 \times 10^{8}$ & $2.2 \times 10^{7}$ & \\
\hline & Urine & & Negative & & Negative & Negative & Negative & \\
\hline & Saliva & & Negative & & $5.26 \times 10^{3}$ & Negative & Negative & \\
\hline \multirow[t]{3}{*}{$17^{*}$} & Blood & & n.d & & n.d & $2.4 \times 10^{9} \dagger$ & n.d & \\
\hline & Urine & & n.d & & n.d & Negative & n.d & \\
\hline & Saliva & & n.d & & n.d & Negative & n.d & \\
\hline \multirow[t]{3}{*}{$20^{*}$} & Blood & & n.d & & n.d & & $1.3 \times 10^{7} \dagger$ & \\
\hline & Urine & & n.d & & n.d & & Negative & \\
\hline & Saliva & & n.d & & n.d & & Negative & \\
\hline \multirow[t]{3}{*}{21} & Blood & & $1.7 \times 10^{9}$ & & $2.0 \times 10^{8}$ & & & \\
\hline & Urine & & Negative & & Negative & & & \\
\hline & Saliva & & Negative & & Negative & & & \\
\hline \multirow[t]{3}{*}{28} & Blood & & $5.4 \times 10^{7}$ & & $4.7 \times 10^{6}$ & & & \\
\hline & Urine & & Negative & & $3.5 \times 10^{4}$ & & & \\
\hline & Saliva & & Negative & & Negative & & & \\
\hline \multirow[t]{3}{*}{$30^{*}$} & Blood & & $7.5 \times 10^{8}$ & & n.d & & & \\
\hline & Urine & & Negative & & n.d & & & \\
\hline & Saliva & & Negative & & n.d & & & \\
\hline \multirow[t]{3}{*}{35} & blood & & $4.4 \times 10^{5}$ & & $7.2 \times 10^{7}$ & & & \\
\hline & Urine & & Negative & & Negative & & & \\
\hline & Saliva & & Negative & & Negative & & & \\
\hline \multirow[t]{3}{*}{$41^{*}$} & Blood & & $9.2 \times 10^{7} \dagger$ & & n.d & & & \\
\hline & Urine & & Negative & & n.d & & & \\
\hline & Saliva & & Negative & & n.d & & & \\
\hline \multirow[t]{3}{*}{42} & Blood & & & & $1.8 \times 10^{7}$ & & & \\
\hline & Urine & & & & Negative & & & \\
\hline & Saliva & & & & Negative & & & \\
\hline \multirow[t]{3}{*}{48} & Blood & & & & $1.4 \times 10^{9}$ & & & \\
\hline & Urine & & & & $2.5 \times 10^{4}$ & & & \\
\hline & Saliva & & & & Negative & & & \\
\hline \multirow[t]{3}{*}{$49^{*}$} & Blood & & & & $1.9 \times 10^{8}$ & & & \\
\hline & Urine & & & & Negative & & & \\
\hline & Saliva & & & & Negative & & & \\
\hline
\end{tabular}


Table 1 (continued)

\begin{tabular}{|c|c|c|c|c|c|c|c|c|}
\hline \multirow{2}{*}{$\begin{array}{l}\text { Days post } \\
\text { infection }\end{array}$} & \multirow[t]{2}{*}{ M. suis $/ \mathrm{mL}$} & \multicolumn{7}{|c|}{ Animal ID (group B animal) } \\
\hline & & ID 23 & ID 31 & ID 32 & ID 71 & ID 73 & ID 74 & ID 76 \\
\hline \multirow[t]{3}{*}{56} & Blood & & & & $1.0 \times 10^{4}$ & & & \\
\hline & Urine & & & & Negative & & & \\
\hline & Saliva & & & & Negative & & & \\
\hline \multirow[t]{3}{*}{$60^{*}$} & Blood & & & & $2.9 \times 10^{9}$ & & & \\
\hline & Urine & & & & Negative & & & \\
\hline & Saliva & & & & Negative & & & \\
\hline \multirow[t]{3}{*}{62} & Blood & & & & $4.9 \times 10^{7} \dagger$ & & & \\
\hline & Urine & & & & Negative & & & \\
\hline & Saliva & & & & Negative & & & \\
\hline
\end{tabular}

Bold lines represent a positive detection of $M$. suis

n.d. not determined

* Additional sampling date due to exacerbation of clinical signs, only clinically affected animals were sampled at this study date

${ }^{\dagger}$ Euthanisation

M. suis loads in blood, saliva and urine samples of group B animals as determined by qPCR are shown in Table 1.

\section{Detection of HMs in blood, urine, saliva and semen in field samples}

Mycoplasma suis was detected in the blood from 61 out of 150 sampled sows while ' $\mathrm{Ca}$. Mycoplasma haemosuis' was detected in 13 out of the 150 samples. Each of the 13 'Ca. M. haemosuis' positive sows were coinfected with M. suis. Blood loads varied from 2.04 to $3.74 \times 10^{7}$ M. suis per $\mathrm{mL}$ blood and from $7.16 \times 10^{3}$ to $4.88 \times 10^{4}$ 'Ca. M haemosuis' per $\mathrm{mL}$ blood, respectively. All 148 corresponding saliva samples (59 samples of HM blood positive sows) revealed negative qPCR results for both HM species (all samples $\mathrm{p}<0.001$, power $100 \%$; samples of bacteremic sows $\mathrm{p}=0.004$, power $100 \%$ ).

Similarly, all 47 urine samples (16 samples of HM blood positive sows) revealed negative qPCR results for both HM species (all samples $\mathrm{p}=0.014$, power $99.8 \%$; samples of blood positive sows $p=0.371$, power $<0.1 \%$ ). $R B C$ residues were not detected in any of the urine samples.

No evidence of $M$. suis and 'Ca. M. haemosuis' infections was detected in any of the 183 tested boars from the boar stud as determined by qPCRs.

\section{Discussion}

Up-to-date, transmission of porcine HMs is thought to mainly occur horizontally during zootechnical procedures and ranking fights. Additionally, results from Heinritzi [7] suggested transmission through oral intake of $M$. suis containing blood. However, oral infection with
M. suis was not proven in our experimental study due to the absence of clinical signs and M. suis in blood samples determined by qPCR throughout the entire study period. Possible explanations for the deviating results might be the different inoculation strains or the higher inoculation dose used by Heinritzi [7] (10 mL containing $5.8 \times 10^{9}-4.17 \times 10^{10} \mathrm{M}$. suis $\left./ \mathrm{mL}\right)$ compared to the present experiment $\left(1.5 \mathrm{~mL} ; 2.0 \times 10^{7} \mathrm{M}\right.$. suis $\left./ \mathrm{mL}\right)$. However, the inoculation dose chosen by Heinritzi [7] does not represent a realistic scenario for field infections as the mean bacterial blood loads found in piglets and sows in previous qPCR field studies were much lower $[13,14]$. Thus, an inoculation dose in accordance with recent studies was chosen in our experiment. Additionally, in contrast to microscopic examination lacking specificity and sensitivity, our results resemble the first investigation of oral transmission routes using up-to-date real-time PCR assays. Similar results were found for the feline HM species 'Ca. M. turicensis' [15] as oral inoculation with ' $\mathrm{Ca}$. M. turicensis' containing blood was not successful in cats.

The detection of $M$. suis in different secretes and excretes of experimentally infected pigs has raised issues on blood independent HM transmission routes [11]. In accordance with this previous study, M. suis could also be detected in urine and saliva samples of group B animals (subcutaneously infected) after experimental infection. Despite comparable M. suis loads in both studies Dietz et al. [11] found a higher number of animals and samples positive for $M$. suis in urine and saliva. Interestingly, in the previous study of Dietz et al. [11] M. suis was also present in urine without $\mathrm{RBC}$ residues whereas all M. suis positive urine samples in our study contained RBC 
residues. Consequently, no $\mathrm{RBC}$-free secretion in urine was observed in the present study. This might be attributable to the variation of the M. suis strain in both studies [11], e.g., the inoculation strain used by Dietz et al. [11] displayed an additional cell tropism for endothelial cells [16].

Regarding samples of naturally infected sows, neither $M$. suis nor ' $\mathrm{C} a$. M. haemosuis' could be identified in urine and saliva samples under field conditions despite the presence of HMs in the corresponding blood samples. This might also be explained by variation of HM strains, higher M. suis blood loads in experimental studies [1012] compared to naturally infected pigs [13, 14], or a higher susceptibility for HM infections due to the splenectomised pig model used for experimental infections [17]. Furthermore, it still has to be scrutinized under experimental conditions if the PCR positive secretes and excretes of the aforementioned experimentally infected animals actually contain infectious organisms.

Despite the very successful application of oral fluidbased testing facilitates for monitoring, surveillance and detection of several pathogens relevant for the swine production [18-24] oral fluids seem not to resemble a suitable diagnostic specimen for the detection of HMs. However, limitations of our study arise from the number of investigated animals and the use of individual swabs that might provide lower detection rates compared to pen-based oral fluids. Therefore, additional studies including pen-based oral fluids with a larger number of animals are warranted to further evaluate the efficacy and sensitivity of HM detection in oral fluids.

The PCR negative blood sample results of the 183 investigated boars from 26 different multiplier farms was somehow unexpected, as previous studies revealed a high prevalence of $M$. suis in sows [25-28]. However, it might be assumed that multiplier farms have a lower risk of $M$. suis introduction due the very limited purchase of animals and strict biosecurity measures.

Semen can serve as an important route for the introduction of various pathogens into a farm [29]. Up-todate, transmission of $M$. suis via semen is thought to occur only in case of blood contamination $[29,30]$. However, those studies were performed in the pre-PCR era and shedding of the pathogen in RBC-free urine, saliva and vaginal secretions has reinforced the discussion of blood-independent transmission route. Under the condition of the present study with investigating samples from one boar stud at one sampling point we were not able to detect porcine HM species in blood and semen. Nevertheless, to exclude boars and semen as potential reservoirs for $\mathrm{HM}$ transmission further studies including a higher number of boar studs and boars from conventional farms are certainly needed.

\section{Conclusion}

In conclusion, our results indicate that blood independent shedding routes are unlikely to play a major epidemiological role under field conditions. In addition, the results of our experimental study did not confirm the possibility of an oral transmission for $M$. suis via infected blood. Despite several benefits over the more invasive blood sampling, individual saliva samples might not represent an appropriate sample type for the detection of $M$. suis.

\section{Materials and methods \\ Experimental design}

For experimental infection, a splenectomised pig model was used [10, 17]. The experimental protocol was approved by the Government Office of Upper Bavaria, Munich (authorization reference number 55.2-1-542532-87-12). A total of 14 piglets at the age of 28-days originating from the same $M$. suis negative farm were included in the study. The $M$. suis negative status was confirmed by qPCR as previously described [13, 31]. One week after placement, each piglet was splenectomised according to the protocol of Heinritzi [17]. For experimental studies with $M$. suis splenectomy is usually performed since the absence of the spleen reduces the incubation period, exacerbates the clinical signs of disease and enhances the replication rate of the pathogen within the host animal [10].

One week after splenectomy, piglets were randomly assigned into two groups (group A: $\mathrm{n}=7$; ID 33, 34, 37, $64,65,55,69$; group B: $\mathrm{n}=7$; ID 23, 31, 32, 71, 73, 74;) for experimental infection. The previously described $M$. suis field strain K323/13 [12] was used as inoculation strain.

Piglets of group A were inoculated orally, piglets of group B subcutaneously with $M$. suis containing blood (1.5 mL; $2.0 \times 10^{7}$.M. suis $\left./ \mathrm{mL}\right)$. Daily clinical observation, treatment and determination of the experiment were performed as previously described [12]. Briefly, the clinical scoring system shown in Table 2 was used for daily observation of animals. Upon acute IAP attack, which is delineated by three clinical score points, animals were treated with oxytetracycline $(20 \mathrm{mg} / \mathrm{kg}$ body weight $/ 24 \mathrm{~h}, \mathrm{i} . \mathrm{m}$.) and glucose ( $35 \mathrm{~g} / \mathrm{L}$, oral). Additionally, Metamizole ( $30 \mathrm{mg} / \mathrm{kg}$ body weight) was administered intramuscularly if the body temperature exceeded $42^{\circ} \mathrm{C}$. The termination criteria of the experiment were defined as follows: a clinical score of $>3$ remaining constant over $48 \mathrm{~h}$ despite antibiotic treatment, sustained fever of $>40{ }^{\circ} \mathrm{C}$ and impaired general health and anorexia. On reaching these criteria, the affected animal was euthanized by intravenous pentobarbital injection $(45 \mathrm{mg} / \mathrm{kg}$ body weight). 
Table 2 Clinical scoring system used for daily animal observation during experimental M. suis infection (in accordance with Stadler et al. [12])

\begin{tabular}{lllllll}
\hline Score points & Ears & Skin & Body temperature & Behavior & Feed intake & Respiration \\
\hline 0 & No alterations & No alterations & $<40{ }^{\circ} \mathrm{C}$ & No alterations & No alterations & No alterations \\
1 & Mild cyanosis & Moderate pallor & $40-42{ }^{\circ} \mathrm{C}$ & Reduced & Reduced & Mild dyspnoe \\
2 & Moderate cyanosis & Generalised petechiae & $>42{ }^{\circ} \mathrm{C}$ & Apathy & Anorexia & Severe dyspnoe \\
3 & and necrosis & & & & - & - \\
\hline
\end{tabular}

EDTA-anticoagulated blood samples (puncture of $V$. jugularis), urine and saliva samples were collected every two days for the first 8 days post infection (DPI) and subsequently once a week until the end of the trial on 90 DPI. Furthermore, individual samples were taken on additional time points when clinical signs exacerbated in the affected animal.

Individual saliva collection was performed without restraining of the animals. Saliva samples were obtained as described elsewhere [32, 33]. Briefly, the pigs were allowed to chew on a cotton swab with the help of a metal rod, until the swab was thoroughly soaked with saliva (Salivette ${ }^{\circledR}$, Sarstedt, Aktiengesellschaft and Company, Nümbrecht, Germany). After sample collection, the swab was placed in a sealed plastic vial and was centrifuged at $4000 \times g$ for $8 \mathrm{~min}$. Urine samples were taken by spontaneous urination in sterile tubes. Saliva and urine samples were stored at $-80^{\circ} \mathrm{C}$ until further processing.

Gross-necropsy and histopathological examination was performed of all animals. as previously described [12]. In brief, tissues were fixed in paraformaldehyde, embedded in plastic and were stained for Giemsa and haematoxyline-eosin-phloxin.

\section{Field samples}

Blood, saliva and urine samples of 150 sows were available from a previous study (stored at $-80{ }^{\circ} \mathrm{C}$ ) [34] (authorization reference number 55.2-154-2532.2-16-13) and collected as described above. The samples originated from 15 piglet producing farms in Southern Germany. The chosen farms were preselected as being positive for $M$. suis by detection of $M$. suis in blood samples of sows by qPCR. In total, 148 saliva samples (8-10 saliva samples and corresponding blood samples per farm) from 15 M. suis positive farms and 47 urine samples (1-8 urine samples and corresponding blood samples per farm) from $11 \mathrm{M}$. suis positive farms were investigated for the presence of $M$. suis by qPCR. A minimum of $10 \%$ positive results were assumed for the statistical analysis (Binomial test and Power calculation), which was calculated with BIAS for Windows 11.01 (Epsilon-Verlag, Frankfurt; Germany).
Additionally, semen and EDTA-blood samples obtained from a German boar stud during regular on-farm health monitoring were also included in the present study. In total semen and EDTA-samples of 183 boars originating from 26 different multiplier farms were collected. The boars' age ranged from 9 to 77 months. Samples were collected within one day and stored at $-80{ }^{\circ} \mathrm{C}$ until further processing. Investigations were approved by the ethical commission of the veterinary faculty of the LMU, Munich (authorization reference number: 245-17-12-2020).

\section{Methods}

DNA was extracted from EDTA-anticoagulated blood, urine, and saliva samples as described previously [11, 13]. Urine samples were further tested with Servotest ${ }^{\circledR}$ $5+$ NL stripes (Servoprax, Wesel, Germany) for RBC residues. Semen samples were pooled to five and DNA was extracted by using the QIAamp ${ }^{\circledR}$ DNA mini kit (Qiagen $\mathrm{GmbH}$, Hilden, Germany) according to the manufacturer's instructions. DNA samples were investigated for $M$. suis and ' $C a$. M. haemosuis' by qPCR as previously described $[6,13,31]$. Briefly, the following primers targeting the msg1 gene of $M$. suis (msg1-Fw $5^{\prime}$-ACAACT AATGCACTAGCTCCTATC-3' and msg1-Rv 5'-GCT CCTGTAGTTGTAGGAATAATTGA) and the gap gene of ' $\mathrm{Ca}$. M. haemosuis' (CMhsuisF 5'-TGCTTTGGC TCCTGTGGTTA-3' and CMhsuisR 5'- GCAGCAGCA CCTGTAG AAGTA-3') were used. The 178 bp fragment ( $M$. suis) and the 177 bp fragment ('Ca. M. haemosuis') were each detected and quantified using the StepOne ${ }^{\mathrm{TM}}$ System (Applied Biosystems ${ }^{\circledR}$ ). QPCR was carried out with Fast SYBR ${ }^{\circledR}$ Green PCR and the following cycling conditions: $95{ }^{\circ} \mathrm{C}$ for $10 \mathrm{~min}$ followed by 40 cycles of $95^{\circ} \mathrm{C}$ for $15 \mathrm{~s}$ and $60{ }^{\circ} \mathrm{C}$ for $30 \mathrm{~s}$ and subsequent melting curve analysis.

\section{Acknowledgements}

Not applicable.

\section{Authors' contributions}

Study conception and design: JS, MR, JA, ME, KH, LEH. Data acquisition: JS, JA, CW, ME. Data analysis and interpretation: JS, JA, SR, CW. Drafting the 
manuscript: JS, JA, KH, LEH. All authors read, critically revised and approved the final manuscript.

\section{Funding}

Open Access funding enabled and organized by Projekt DEAL.

\section{Availability of data and materials}

All datasets used in this study are available from the corresponding author on reasonable request.

\section{Declarations}

\section{Ethics approval and consent to participate}

The protocol for experimental infection as well as all procedures were officially approved by the Government Office of Upper Bavaria, Munich, Germany (authorization reference number 55.2-1-54-2532-87-12). The study was compliant with all relevant European guidelines and regulations for animal experiments. The study was carried out in compliance with the ARRIVE guidelines. Collection of field samples from sows were also approved approved by the Government Office of Upper Bavaria, Munich, Germany (authorization reference number 55.2-154-2532.2-16-13). Sampling of the boars was approved by the ethical commission of the veterinary faculty of the LMU, Munich (authorization reference number: 245-17-12-2020).

\section{Consent for publication}

Not applicable.

\section{Competing interests}

The authors declare that they have no competing interests.

\section{Author details}

${ }^{1}$ Clinic for Swine, Centre for Clinical Veterinary Medicine, LMU Munich, Sonnenstr. 16, Oberschleissheim, 85764 Munich, Germany. ${ }^{2}$ Institute of Animal Science, University of Hohenheim, Stuttgart, Germany. ${ }^{3}$ Institute for Anatomy, Histology and Embryology, LMU Munich, Munich, Germany.

Received: 3 May 2021 Accepted: 9 August 2021

Published online: 26 August 2021

\section{References}

1. Messick JB. Hemotrophic mycoplasmas (hemoplasmas): a review and new insights into pathogenic potential. Vet Clin Pathol. 2004;33(1):2-13.

2. Hoelzle LE, Zeder M, Felder KM, Hoelzle K. Pathobiology of Mycoplasma suis. Vet J. 2014;202(1):20-5.

3. Hoelzle LE, Felder KM, Hoelzle K. Porcine eperythrozoonosis: from Eperythrozoon suis to Mycoplasma suis. Tierarztl Prax Ausg G Grosstiere Nutztiere. 2011;39(4):215-20.

4. Fu Y, Shi T, Xu L, Wei W, Lu F, Zhang X, et al. Identification of a novel Hemoplasma species from pigs in Zhejiang province. China J Vet Med Sci. 2017:79(5):864-70.

5. Seo MG, Kwon OD, Kwak D. Prevalence and phylogenetic analysis of hemoplasma species in domestic pigs in Korea. Parasit Vectors. 2019;12(1):378.

6. Stadler J, Ade J, Ritzmann M, Hoelzle K, Hoelzle LE. Detection of a novel haemoplasma species in fattening pigs with skin alterations, fever and anaemia. Vet Rec. 2020;187(2):66.

7. Heinritzi K. Untersuchungen zur Übertragung von Eperythrozoon suis. Tieraertzliche Umschau. 1992;47:588-99.

8. Prullage JB, Williams RE, Gaafar SM. On the transmissibility of Eperythrozoon suis by Stomoxys calcitrans and Aedes aegypti. Vet Parasitol. 1993:50(1-2):125-35

9. Schwarz L, Strauss A, Loncaric I, Spergser J, Auer A, Rümenapf T, et al. The Stable Fly (Stomoxys calcitrans) as a Possible Vector Transmitting Pathogens in Austrian Pig Farms. Microorganisms. 2020;8(10).

10. Stadler J, Jannasch C, Mack SL, Dietz S, Zöls S, Ritzmann M, et al. Clinical and haematological characterisation of Mycoplasma suis infections in splenectomised and non-splenectomised pigs. Vet Microbiol. 2014;172(1-2):294-300.

11. Dietz S, Mack SL, Hoelzle K, Becker K, Jannasch C, Stadler J, et al. Quantitative PCR analysis of Mycoplasma suis shedding patterns during experimental infection. Vet Microbiol. 2014;172(3-4):581-5.

12. Stadler J, Ade J, Hermanns W, Ritzmann M, Wentzel S, Hoelzle K, et al. Clinical, haematological and pathomorphological findings in Mycoplasma suis infected pigs. BMC Vet Res. 2021;17(1):214.

13. Stadler J, Willi S, Ritzmann M, Eddicks M, Ade J, Hoelzle K, et al. Detection of Mycoplasma suis in pre-suckling piglets indicates a vertical transmission. BMC Vet Res. 2019;15(1):252.

14. Ritzmann M, Grimm J, Heinritzi K, Hoelzle K, Hoelzle LE. Prevalence of Mycoplasma suis in slaughter pigs, with correlation of PCR results to hematological findings. Vet Microbiol. 2009;133(1-2):84-91.

15. Museux K, Boretti FS, Willi B, Riond B, Hoelzle K, Hoelzle LE, et al. In vivo transmission studies of "Candidatus Mycoplasma turicensis" in the domestic cat. Vet Res. 2009;40(5):45.

16. Sokoli A, Groebel K, Hoelzle K, Amselgruber WM, Mateos JM, Schneider MK, et al. Mycoplasma suis infection results endothelial cell damage and activation: new insight into the cell tropism and pathogenicity of hemotrophic mycoplasma. Vet Res. 2013;44(1):6.

17. Heinritzi K. A contribution on splenectomy in swine. Tierarztl Prax. 1984;12(4):451-4.

18. Grau FR, Schroeder ME, Mulhern EL, Mclntosh MT, Bounpheng MA. Detection of African swine fever, classical swine fever, and footand-mouth disease viruses in swine oral fluids by multiplex reverse transcription real-time polymerase chain reaction. J Vet Diagn Invest. 2015;27(2):140-9.

19. Senthilkumaran C, Yang M, Bittner H, Ambagala A, Lung O, Zimmerman $J$, et al. Detection of genome, antigen, and antibodies in oral fluids from pigs infected with foot-and-mouth disease virus. Can J Vet Res. 2017;81(2):82-90.

20. Goodell CK, Prickett J, Kittawornrat A, Zhou F, Rauh R, Nelson W, et al. Probability of detecting influenza A virus subtypes H1N1 and H3N2 in individual pig nasal swabs and pen-based oral fluid specimens over time. Vet Microbiol. 2013;166(3-4):450-60.

21. Decorte I, Van Campe W, Mostin L, Cay AB, De Regge N. Diagnosis of the Lelystad strain of Porcine reproductive and respiratory syndrome virus infection in individually housed pigs: comparison between serum and oral fluid samples for viral nucleic acid and antibody detection. J Vet Diagn Invest. 2015;27(1):47-54.

22. Prickett JR, Johnson J, Murtaugh MP, Puvanendiran S, Wang C, Zimmerman JJ, et al. Prolonged detection of PCV2 and anti-PCV2 antibody in oral fluids following experimental inoculation. Transbound Emerg Dis. 2011;58(2):121-7.

23. Bjustrom-Kraft J, Woodard K, Giménez-Lirola L, Rotolo M, Wang C, Sun $Y$, et al. Porcine epidemic diarrhea virus (PEDV) detection and antibody response in commercial growing pigs. BMC Vet Res. 2016;12:99.

24. Panyasing Y, Kedkovid R, Kittawornrat A, Ji J, Zimmerman J, Thanawongnuwech R. Detection of Aujeszky's disease virus DNA and antibody in swine oral fluid specimens. Transbound Emerg Dis. 2018;65(6):1828-35.

25. Gatto IRH, Sonálio K, Amaral RBD, Morés N, Dalla Costa OA, André MR, et al. High frequency and molecular characterization of porcine hemotrophic mycoplasmas in Brazil. Vet Microbiol. 2019;231:33-9.

26. Song Q, Zhang W, Song W, Liu Z, Khan MK, He L, et al. Seroprevalence and risk factors of Mycoplasma suis infection in pig farms in central China. Prev Vet Med. 2014;117(1):215-21.

27. Zhongyang $L$, Jiansong $Z$, Yijuan $S$, Yuting $X$, Yufeng $L$, Jiarong $X$. Seroprevalence of Mycoplasma suis infection in pigs in eastern China as estimated by a blocking enzyme-linked immunosorbent assay. Can J Vet Res. 2017:81(4):313-7.

28. Brissonnier M, Normand V, Lebret A, Moalic PY, Guyomard AS, Bachy V, et al. Frequency of infection with Mycoplasma suis in gestating sows using $\mathrm{QPCR}$ on ten commercial French herds, and impact of the infection on clinical, haematological and biochemical parameters. Porcine Health Manag. 2020;6:13.

29. Maes D, Nauwynck H, Rijsselaere T, Mateusen B, Vyt $P$, de Kruif A, et al. Diseases in swine transmitted by artificial insemination: an overview. Theriogenology. 2008;70(8):1337-45 
30. Heinritzi K. Eperythrozoonosis. In: Straw B, D'Allaire S, Mengeling W, Taylor D, editors. Diseases of Swine 8th ed: Ames IA, USA: Iowa State University Press; 1999. p. 413-8.

31. Hoelzle LE, Helbling M, Hoelzle K, Ritzmann M, Heinritzi K, Wittenbrink MM. First LightCycler real-time PCR assay for the quantitative detection of Mycoplasma suis in clinical samples. J Microbiol Methods. 2007;70(2):346-54

32. Gutiérrez AM, Martínez-Subiela S, Cerón JJ. Evaluation of an immunoassay for determination of haptoglobin concentration in various biological specimens from swine. Am J Vet Res. 2009;70(6):691-6.

33. Gutiérrez AM, Martínez-Subiela S, Eckersall PD, Cerón JJ. C-reactive protein quantification in porcine saliva: a minimally invasive test for pig health monitoring. Vet J. 2009;181(3):261-5
34. Eddicks M, Koeppen M, Willi S, Fux R, Reese S, Sutter G, et al. Low prevalence of porcine circovirus type 2 infections in farrowing sows and corresponding pre-suckling piglets in southern German pig farms. Vet Microbiol. 2016;187:70-4.

\section{Publisher's Note}

Springer Nature remains neutral with regard to jurisdictional claims in published maps and institutional affiliations.
Ready to submit your research? Choose BMC and benefit from:

- fast, convenient online submission

- thorough peer review by experienced researchers in your field

- rapid publication on acceptance

- support for research data, including large and complex data types

- gold Open Access which fosters wider collaboration and increased citations

- maximum visibility for your research: over $100 \mathrm{M}$ website views per year

At BMC, research is always in progress.

Learn more biomedcentral.com/submissions 